Check for updates

Cite this: New J. Chem., 2021, 45, 3892

Received 8th December 2020, Accepted 25th January 2021

DOI: $10.1039 / d 0 n j 06000 c$

rsc.li/njc

\title{
Hydrogenation and oxidation enhances the thermoelectric performance of $\mathrm{Si}_{2} \mathrm{BN}$ monolayer
}

\author{
H. R. Mahida, ${ }^{a}$ Deobrat Singh, (DD*b ${ }^{b}$ Yogesh Sonvane, (D) ${ }^{c}$ Sanjeev K. Gupta, (D) ${ }^{d}$ \\ P. B. Thakor ${ }^{\star a}$ and Rajeev Ahuja (D) be
}

In the present study, we have investigated the structural, electronic, and charge transport properties of pristine, hydrogenated, and oxidized $\mathrm{Si}_{2} \mathrm{BN}$ monolayers via first-principles calculations based on density functional theory (DFT). Hydrogenation and oxidation of $\mathrm{Si}_{2} \mathrm{BN}$ monolayer display negative binding energy therefore these structures are energetically favorable. The electronic band structure engineered by the hydrogenation and oxidation of the $\mathrm{Si}_{2} \mathrm{BN}$ monolayer transformed from metallic to semiconducting nature. Due to the hydrogenation and oxidation of $\mathrm{Si}_{2} \mathrm{BN}$, the monolayer also changes from a planar structure to a non-planar structure. The hydrogenated and oxidized structures led to high thermoelectric performance as compared to the pristine $\mathrm{Si}_{2} \mathrm{BN}$ monolayer. When the $\mathrm{Si}_{2} \mathrm{BN}$ monolayer is hydrogenated and oxidized, its electronic figure of merit $\left(Z T_{\mathrm{e}}\right)$ significantly enhanced from 0.45 to 0.99 . The investigation results suggest a practical approach for improving the performance of thermoelectric properties of the $\mathrm{Si}_{2} \mathrm{BN}$ monolayer.

\section{Introduction}

Thermoelectricity has seen a revival of late due to the perceived dangers of fossil fuels and global warming. Although the phenomenon has been known since the 19th century, thermoelectric (TE) materials have recently attracted keen interest. ${ }^{1}$ As an essential carrier for energy conversion between heat and electricity, thermoelectric materials have been widely studied and considerable progress has been made in the past several decades. Thermoelectric materials can generate power via the Seebeck effect or refrigerate using the Peltier effect. They are capable of converting heat flow directly into electrical energy or vice versa. ${ }^{2,3}$ Thermoelectric materials can be employed in aerospace applications, solar energy, cooling-thermal management, waste heat recovery, and electronic techniques due to the characteristics of being scalable, lightweight, pollutionfree, having no moving parts and long operating life. ${ }^{4-6}$ The development of new, more efficient materials and devices

\footnotetext{
${ }^{a}$ Department of Physics, Veer Narmad South Gujarat University, Surat 395007, India. E-mail: pbthakor@rediffmail.com

${ }^{b}$ Condensed Matter Theory Group, Materials Theory Division, Department of Physics and Astronomy, Uppsala University, Box 516, 75120, Uppsala, Sweden. E-mail: deobrat.singh@physics.uu.se

${ }^{c}$ Advanced Material Lab, Department of Physics, Sardar Vallabhbhai National Institute of Technology, Surat 395007, India

${ }^{d}$ Computational Materials and Nanoscience Group, Department of Physics and Electronics, St. Xavier's College, Ahmedabad 380009, India

${ }^{e}$ Applied Materials Physics, Department of Materials Science and Engineering, Royal Institute of Technology (KTH), S-100 44 Stockholm, Sweden
}

is the key to expand the range of thermoelectric generators and coolers. ${ }^{7}$

In general, thermoelectric efficiency is determined by the dimensionless figure of merit, $Z T$, defined as $Z T=S^{2} T \sigma / k$, where $S, T, \sigma$ and $k$ are Seebeck coefficient, absolute temperature, electrical conductivity, and thermal conductivity, respectively. Thermal conductivity contains both the lattice and electronic parts; $k=k_{1}+k_{\mathrm{e}}$, where, $k_{1}$ is the lattice thermal conductivity relative to lattice vibrations (phonon), and $k_{\mathrm{e}}$ is the electronic thermal conductivity related to the electronic structure. Over the last many years, intensive research has been done to increase $Z T$ for high thermoelectric performance. ${ }^{8-17}$ It was previously reported that graphene and boron nitride have very high formation energy. The formation energy of graphene is $-84.95 \mathrm{eV}$ and that of monolayer boron nitrate is $-81.70 \mathrm{eV} .^{1}$ Motivated by this concern, we found the formation energy of $-50.38 \mathrm{eV}$ for the $\mathrm{Si}_{2} \mathrm{BN}$ single layer. Because of its $2 \mathrm{D}$ nature, the $\mathrm{Si}_{2} \mathrm{BN}$ monolayer is flexible and strong. It could be similar other properties of $2 \mathrm{D}$ materials that indicate superior and high electron flexibilities with tunable electronic band structure and high thermal conductivities. Moreover, the presence of $\mathrm{Si}$ atoms in the monolayer of $\mathrm{Si}_{2} \mathrm{BN}$ will make the surface more reactive. Therefore, it is a very interesting candidate material for hydrogen storage. ${ }^{2-8}$ In all of these materials, such as 2D graphene, the $\mathrm{Si}_{2} \mathrm{BN}$ monolayer has evoked considerable attention for its potential use and application in hydrogen storage. ${ }^{9-12}$

We focus on the $\mathrm{Si}_{2} \mathrm{BN}$ monolayer in the present work and investigate the correlation between structural, electronic structure, 
and thermoelectric properties of pristine, hydrogenated, and oxidized monolayer of $\mathrm{Si}_{2} \mathrm{BN}$. The hydrogen and oxygen atoms can prevent the effect of surface states. The simulations of thermoelectric properties via first-principles calculations are based on the density functional theory and Boltzmann transport approach. The computational details are relevant to the structural properties, electronic properties, electronic band structures, and projected density of pristine, hydrogenated, and oxidized $\mathrm{Si}_{2} \mathrm{BN}$ monolayer states.

\section{Computational methods}

In this study, density functional theory calculations were performed using projected augmented wave (PAW) potential, with the generalized gradient approximation (GGA) in the form of Perdew-Burke-Ernzerhof (PBE) functional for the electronic exchange-correlation function, ${ }^{18,19}$ which is implemented in the Vienna Ab initio Simulation Package (VASP) code. ${ }^{20-22}$ An energy cut-off for a plane wave basis set was set to be $500 \mathrm{eV}$, and Brillion Zone (BZ) sampling with the Monkhorst-Pack $(\mathrm{MP})^{23}$ method of $21 \times 21 \times 1 k$-points were selected for the pristine $\mathrm{Si}_{2} \mathrm{BN}$ monolayer, hydrogenated and oxidized $\mathrm{Si}_{2} \mathrm{BN}$ monolayer, respectively. Atomic structures were shown using the VESTA package, ${ }^{24}$ and the HSE06 functional ${ }^{25}$ was used for accurate electronic structure calculations. The band structure was calculated using the HSE06 functional based on the optimized geometrical structures. For the hybrid functional, we have used the exchange-correlation energy functional HSE06 with a mixing parameter $(\alpha)$ of $25 \%$ and a screening parameter of $0.2 \AA^{-1}$. Electronic transport coefficients were computed by solving the semi-classical Boltzmann transport equation (BTE) within the constant relaxation time approximation implemented in the BoltzTraP Program. ${ }^{26} \mathrm{~A}$ constant relaxation time $(\tau)$ with $10^{-14} \mathrm{~s}$ has been used in the thermoelectric calculations.

\section{Results and discussion}

\section{Structural properties}

Fig. 1 shows that the relaxed structure of the graphene-like pristine $\mathrm{Si}_{2} \mathrm{BN}\left(\mathrm{PSi}_{2} \mathrm{BN}\right)$ with the unit cell having the hexagonal symmetry and planar structure. The optimized bond length of $\mathrm{PSi}_{2} \mathrm{BN}$ is $1.46 \AA$ between $\mathrm{B}-\mathrm{N}$ atoms, $2.24 \AA$ between $\mathrm{Si}-\mathrm{Si}$ atoms, $1.75 \AA$ between $\mathrm{Si}-\mathrm{N}$ atoms, and $1.95 \AA$ between $\mathrm{Si}-\mathrm{B}$ atoms. We obtained the optimized lattice constants: $a=6.35 \AA$, $b=6.45 \AA$ and $c=20 \AA$ for $\mathrm{PSi}_{2} \mathrm{BN}$, in accordance with the previous work. ${ }^{27-29}$ Fig. 1 displays the compound of boron, nitrogen, and silicon, which are planarly stabilized and strongly bonded. Due to the higher formation energy, the $\mathrm{PSi}_{2} \mathrm{BN}$ monolayer has a more stable structure. ${ }^{27,30}$ In the previous study, the $\mathrm{Si}_{2} \mathrm{BN}$ monolayer was found to be energetically, thermally and dynamically stable, which was confirmed by the formation energy, ab initio molecular dynamics (AIMD) and phonon calculations. ${ }^{30}$
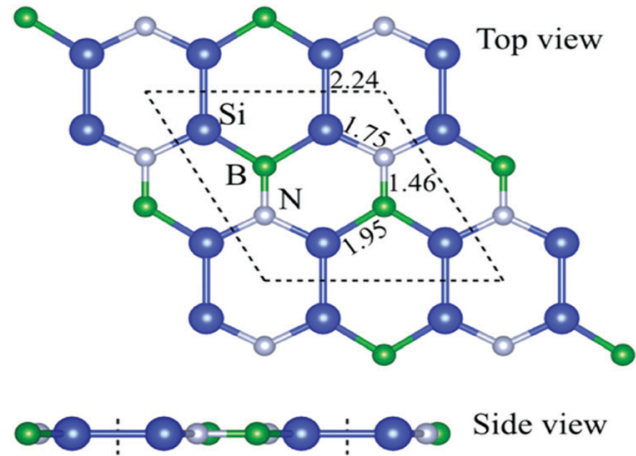

Fig. 1 Optimized structure of the pristine $\mathrm{Si}_{2} \mathrm{BN}$ monolayer. The dashed line denotes the unit cell of $\mathrm{Si}_{2} \mathrm{BN}$. Blue, green, and silver balls represent $\mathrm{Si}$, $\mathrm{B}$, and $\mathrm{N}$ atoms, respectively.

Also, Fig. 2a-c show the relaxed structure of the hydrogenated $\mathrm{Si}_{2} \mathrm{BN}$ surface by full hydrogenation, hydrogenation of the Si atom on both sides, and hydrogenation of each atom on a single side, respectively. Fig. $2 \mathrm{~b}$ and $\mathrm{c}$ shows the top view of hydrogenation of the $\mathrm{Si}$ atom on both sides and hydrogenation of each atom on a single side, respectively. During full hydrogenation, the bond length changed slightly, with the corresponding bond lengths changing from 2.24 to $2.30 \AA$ \& 1.95 to $2.12 \AA, 1.75$ to $1.82 \AA$, 1.46 to $1.54 \AA$, while in the case of hydrogenation of the $\mathrm{Si}$ atom on both sides, the bond lengths changed from 2.24 to $2.31 \AA$, 1.95 to $2.02 \AA, 1.75$ to $1.80 \AA$, 1.46 to $1.34 \AA$, respectively. Also, in the case of hydrogenation of each atom on a single side, the bond length changed slightly, with the corresponding bond lengths changing from 2.24 to $2.31 \AA, 1.95$ to $1.93 \AA, 1.75$ to $1.82 \AA$, and 1.46 to $1.56 \AA$.

Moreover, Fig. $2 \mathrm{~d}-\mathrm{f}$ represent the relaxed structure of the oxidized $\mathrm{Si}_{2} \mathrm{BN}$ surface after full oxidation, oxidation of the $\mathrm{Si}$ atom on both sides, and hydrogenation of each atom on a single side, respectively. In the case of oxidation of the $\mathrm{Si}_{2} \mathrm{BN}$ monolayer, we have noticed that the bond length slightly changed with corresponding bond lengths changing from 2.24 to $2.86 \AA, 1.95$ to $1.95 \AA, 1.75$ to $1.66 \AA, 1.46$ to $1.40 \AA$, while in the case of oxidation of the Si atom on both sides, the corresponding bond lengths change from 2.24 to $2.29 \AA$, 1.95 to $2.40 \AA, 1.75$ to $1.71 \AA$, 1.46 to $1.51 \AA$. Also, the oxidation of the $\mathrm{Si}_{2} \mathrm{BN}$ monolayer on a single side changed the corresponding bond lengths from 2.24 to $2.30 \AA$, 1.95 to $2.06 \AA$, 1.75 to $1.74 \AA$, and 1.46 to $1.48 \AA$.

To calculate the possibility of achieving the experimental synthesis and the $\mathrm{Si}_{2} \mathrm{BNH}$ and stability of the $\mathrm{Si}_{2} \mathrm{BNO}$ monolayers, we first calculated its cohesive energy, $E_{\mathrm{coh}}$, which is defined by:

$$
E_{\mathrm{coh}}=\frac{\left(E_{\mathrm{Si}_{2} \mathrm{BN}(\mathrm{H} / \mathrm{O})}-n_{\mathrm{Si}} E_{\mathrm{Si}}-n_{\mathrm{B}} E_{\mathrm{B}}-n_{\mathrm{N}} E_{\mathrm{N}}-n_{\mathrm{H} / \mathrm{O}} E_{\mathrm{H} / \mathrm{O}}\right)}{\left(n_{\mathrm{Si}}+n_{\mathrm{B}}+n_{\mathrm{N}}+n_{\mathrm{H} / \mathrm{O}}\right)},
$$

where $E_{\mathrm{Si} 2 \mathrm{BN}(\mathrm{H} / \mathrm{O})}, E_{\mathrm{Si}}, E_{\mathrm{B}}, E_{\mathrm{N}}$, and $E_{\mathrm{H} / \mathrm{O}}$ are the calculated total energies of the hydrogenated/oxidized $\mathrm{Si}_{2} \mathrm{BN}$ monolayer, the total energy of isolated $\mathrm{Si}, \mathrm{B}, \mathrm{N}$, and $\mathrm{H} / \mathrm{O}$ atoms, respectively. $n_{\mathrm{Si}}, n_{\mathrm{B}}, n_{\mathrm{N}}$, and $n_{\mathrm{H} / \mathrm{O}}$ are the numbers of $\mathrm{Si}, \mathrm{B}, \mathrm{N}$, and $\mathrm{H} / \mathrm{O}$ atoms 
(a)

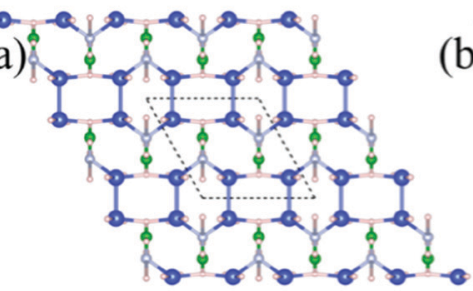

(b)

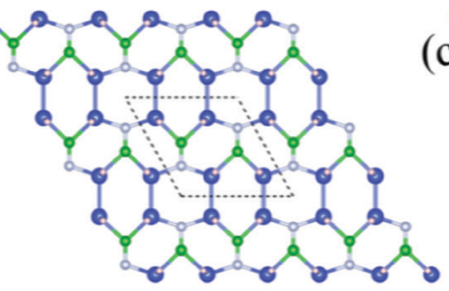

(c)

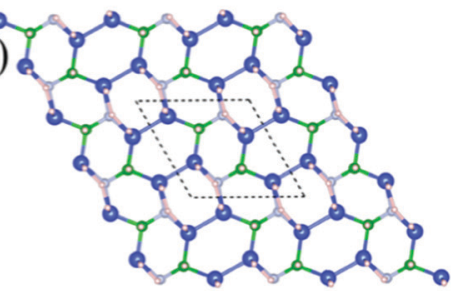

(d)

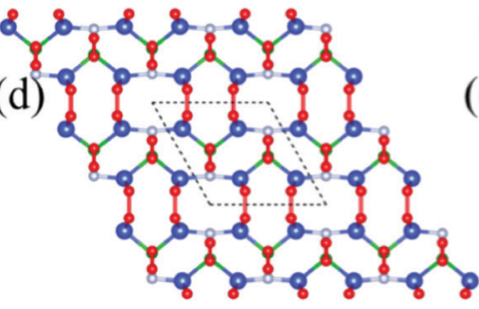

(e)

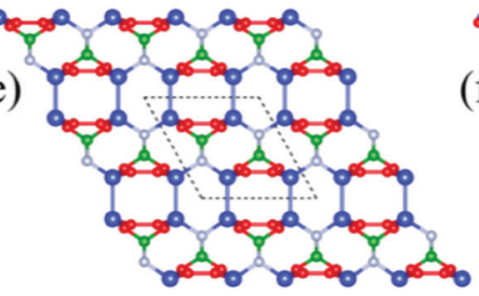

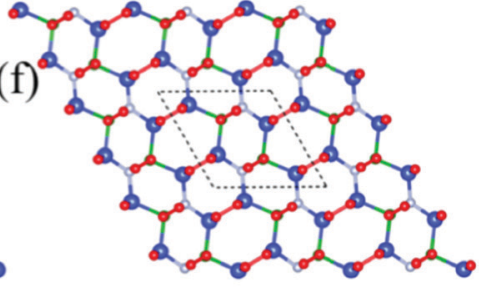

Fig. 2 Optimized structure after the hydrogenation and oxidation of the $\mathrm{Si}_{2} \mathrm{BN}$ surface, (a) full hydrogenation ( $\left.\mathrm{Si}_{2} \mathrm{BNH}\right)$, (b) hydrogenation of the $\mathrm{Si}$ atom on both sides $\left(\mathrm{Si}_{2} \mathrm{BNH}_{\mathrm{si}}\right)$, (c) hydrogenation of each atom on a single side $\left(\mathrm{Si}_{2} \mathrm{BNH}_{\mathrm{s}}\right)$, (d) full oxidation ( $\left.\mathrm{Si}_{2} \mathrm{BNO}\right)$, (e) oxidation of the $\mathrm{Si}$ atom on both sides $\left(\mathrm{Si}_{2} \mathrm{BNO}_{\mathrm{Si}}\right)$, (f) oxidation of each atom on a single side $\left(\mathrm{Si}_{2} \mathrm{BNO}_{\mathrm{S}}\right)$. The dashed line denotes the unit cell of $\mathrm{Si}_{2} \mathrm{BN}$. Blue, green, silver, light pink and red balls represent $\mathrm{Si}, \mathrm{B}, \mathrm{N}, \mathrm{H}$, and $\mathrm{O}$ atoms, respectively.

in the unit cell of the $\mathrm{Si}_{2} \mathrm{BN}$ monolayer, respectively. The computed cohesive energies are $-2.0,-1.38,-1.43 \mathrm{eV}$ per atom for hydrogenation for $\mathrm{Si}_{2} \mathrm{BNH}, \mathrm{Si}_{2} \mathrm{BNH}_{\mathrm{Si}}$ and $\mathrm{Si}_{2} \mathrm{BNH}_{\mathrm{S}}$, respectively, and $-3.28,-3.56,-2.53 \mathrm{eV}$ per atom for oxidation for $\mathrm{Si}_{2} \mathrm{BNO}, \mathrm{Si}_{2} \mathrm{BNO}_{\mathrm{Si}}$, and $\mathrm{Si}_{2} \mathrm{BNO}_{\mathrm{S}}$, respectively. This value is larger than that of the already-synthesized ${ }^{31}$ antimonene $(\sim 2.80 \mathrm{eV}$ per atom $),{ }^{32}$ silicene $(3.95 \mathrm{eV}$ per atom $),{ }^{33}$ arsenene $(2.95 \mathrm{eV} \text { per atom })^{34}$ and phosphorene $(3.48 \mathrm{eV}$ per atom $),{ }^{35}$ indicating the high stability and synthetic feasibility of the hydrogenation and oxidation of the $\mathrm{Si}_{2} \mathrm{BN}$ monolayer from the perspective of energy level.

\section{Electronic properties}

The electronic band structure and partial density of states (PDOS) are represented in Fig. 3. The electronic band structure shows the metallic behavior of $\mathrm{PSi}_{2} \mathrm{BN}$. Despite the significant reduction in symmetry, $\mathrm{PSi}_{2} \mathrm{BN}$ still retains the inversion symmetry that causes the metallic nature. Here, PDOS is measured to understand the orbital contribution at Fermi level $E_{\mathrm{F}}$; the main contribution to the states at $E_{\mathrm{F}}$ comes from the p states of
' $\mathrm{Si}$ ', 'B', and ' $\mathrm{N}$ ' atoms. The contribution of the $\mathrm{p}$ orbital of $\mathrm{Si}$ is much higher than that of the $\mathrm{p}$ orbital of ' $\mathrm{B}$ ' and ' $\mathrm{N}$ ' atoms. The contribution of s-orbital of $\mathrm{Si}, \mathrm{B}$ and $\mathrm{N}$ atoms is noticeable near the Fermi level. The presence of electronic states is found at the Fermi level, indicating the conducting behavior. Fig. 3b shows the decomposed electronic band structure of $\mathrm{PSi}_{2} \mathrm{BN}$ in which we can see that the p orbitals of $\mathrm{Si}$ and B atoms are strongly hybridized, and a small contribution comes from the $\mathrm{p}$ orbital of the $\mathrm{N}$ atom. In addition, one of the electronic states of the valence band maxima crosses the Fermi level, thus showing the conducting nature.

Fig. 4 shows the electronic band structure, projected density of states, and corresponding decomposed band structure of the $\mathrm{Si}_{2} \mathrm{BNH}$ monolayer. The band structure is a semiconductor with a very small bandgap of $0.08 \mathrm{eV}$ with a direct gap at the $\Gamma$ point (see Fig. 4a). Furthermore, the s orbitals of $\mathrm{H}$ atoms are strongly hybridized with the $\mathrm{p}$ orbitals of $\mathrm{Si}, \mathrm{B}$, and $\mathrm{N}$ atoms near the Fermi level, as shown in Fig. 4b. The clear hybridization of each atom can be seen in the decomposed electronic band structure, as depicted in Fig. 4c. Moreover, when the $\mathrm{H}$ atom is attached
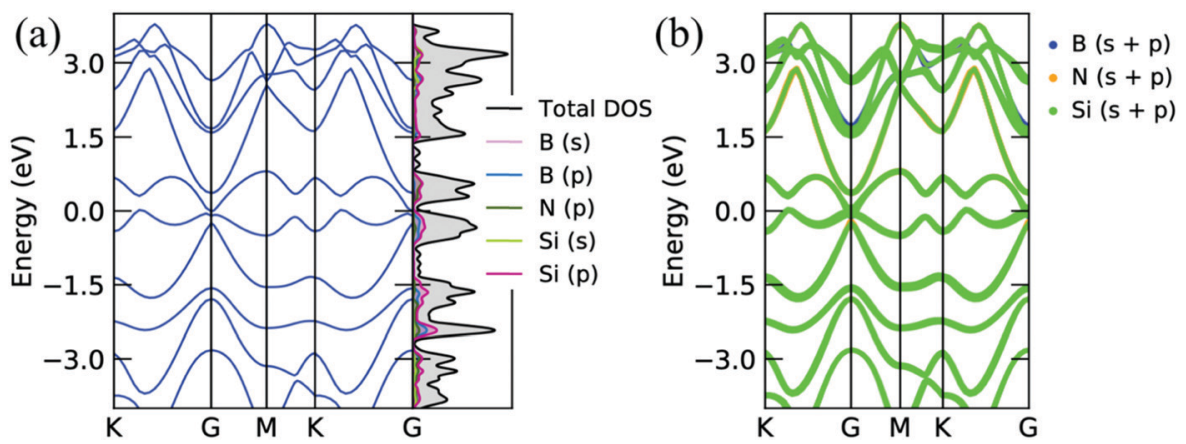

Fig. 3 (a) Electronic band structures and corresponding projected density of pristine $\mathrm{Si}_{2} \mathrm{BN}$ monolayer states. (b) Decomposed electronic band structure of the pristine $\mathrm{Si}_{2} \mathrm{BN}$ monolayer. 

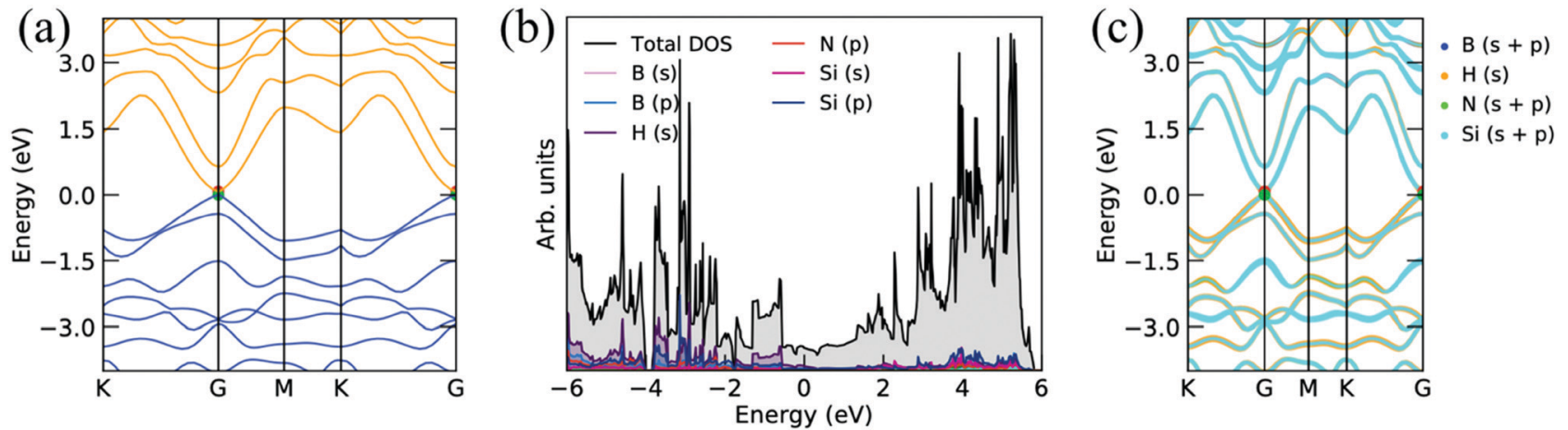

Fig. 4 Electronic band structures and corresponding projected density of states of the $\mathrm{Si}_{2} \mathrm{BN}$ surface with full hydrogenation

to the single side of the $\mathrm{Si}_{2} \mathrm{BN}$ monolayer, the valence and conduction bands are separated by $1.14 \mathrm{eV}$. It means that the $\mathrm{Si}_{2} \mathrm{BNH}_{\mathrm{S}}$ monolayer exhibits the semiconducting behavior. The electronic band gap appears between $K$ to $\Gamma$ point (indirect band gap). From the projected density of states and decomposed band structures, we can say that the s orbital of the $\mathrm{H}$ atom is strongly hybridized with the porbitals of the Si atom near the Fermi level in the valence band (see Fig. $5 \mathrm{~b}$ and c).

The electronic band structure presented in Fig. 5 a has a flat band at VBM, and the flat band has a large effective mass. Thus, large thermo power interprets large effective mass because thermo power is directly proportional to the effective mass, i.e., $S \propto m^{*}$ (also, carrier mobility is inversely proportional to the effective mass, i.e., $\mu \propto 1 / m^{*}$ ), which is equivalent to the large density-of-states or flat bands at the Fermi level. Accordingly, the large effective mass reduces the electric conductivity. In addition, the electronic band structures show semiconductor behavior with a small band gap of $0.29 \mathrm{eV}$ when $\mathrm{H}$ atoms are attached to the $\mathrm{Si}$ atoms on both sides of the $\mathrm{Si}_{2} \mathrm{BN}$ monolayer, as presented in Fig. 6. From Fig. $6 \mathrm{~b}$ and c, we can see that the $\mathrm{s}$ orbital of the $\mathrm{H}$ atom and $\mathrm{p}$ orbital of the $\mathrm{Si}$ atom are more dominating at $E_{\mathrm{F}}$ in VBM, and a small contribution comes from other atoms. It is also found that the flat band and a large number of states near $E_{\mathrm{F}}$ enhance the thermo power.

Furthermore, we have discussed the electronic properties of the oxidized surface of the $\mathrm{Si}_{2} \mathrm{BN}$ monolayer, as presented in Fig. 7-9. A large number of electronic states are present at the $E_{\mathrm{F}}$, which are responsible for enhancing the thermo power, as shown in the electronic band structure (see Fig. 7). The p-orbital of the $\mathrm{O}$ atom is responsible for improving the electronic states at $E_{\mathrm{F}}$, as presented in the projected density of states and decomposed band structure (see Fig. $7 \mathrm{~b}$ and c). The relatively flat band also appeared at the top of the valence band, enhancing the thermo power, as discussed in the below section. Similar types of electronic band structures are found in $\mathrm{O}$ atoms attached to the single side of the $\mathrm{Si}_{2} \mathrm{BN}$ monolayer (see Fig. 8), while it has relatively low flat band lines at the top of the conduction band. It means that the $\mathrm{Si}_{2} \mathrm{BNO}_{\mathrm{S}}$ monolayer has a relatively low thermo power as compared to the $\mathrm{Si}_{2} \mathrm{BNO}$ monolayer. From the PDOS and decomposed band structure, we notice that a significant contribution comes from 'O' atom at $E_{\mathrm{F}}$ compared to other atoms.

Furthermore, $\mathrm{O}$ attached with $\mathrm{Si}$ atoms on both sides of the $\mathrm{Si}_{2} \mathrm{BN}$ monolayer shows a semiconducting behavior with a higher band gap (indirect) of $2.10 \mathrm{eV}$, as depicted in Fig. 9 . The contribution of the $\mathrm{p}$ orbital of the ' $\mathrm{O}$ ' atom is more dominant at the Fermi level than the p-orbitals of 'Si' and ' $N$ ' atoms and s-orbitals of 'Si' and ' $\mathrm{O}$ ' atoms, as presented in PDOS and decomposed band structure (see Fig. $9 \mathrm{~b}$ and c). It also has a sharp peak of electronic states at $E_{\mathrm{F}}$ that is why thermo power is relatively higher than the pristine $\mathrm{Si}_{2} \mathrm{BN}$ monolayer. The effect
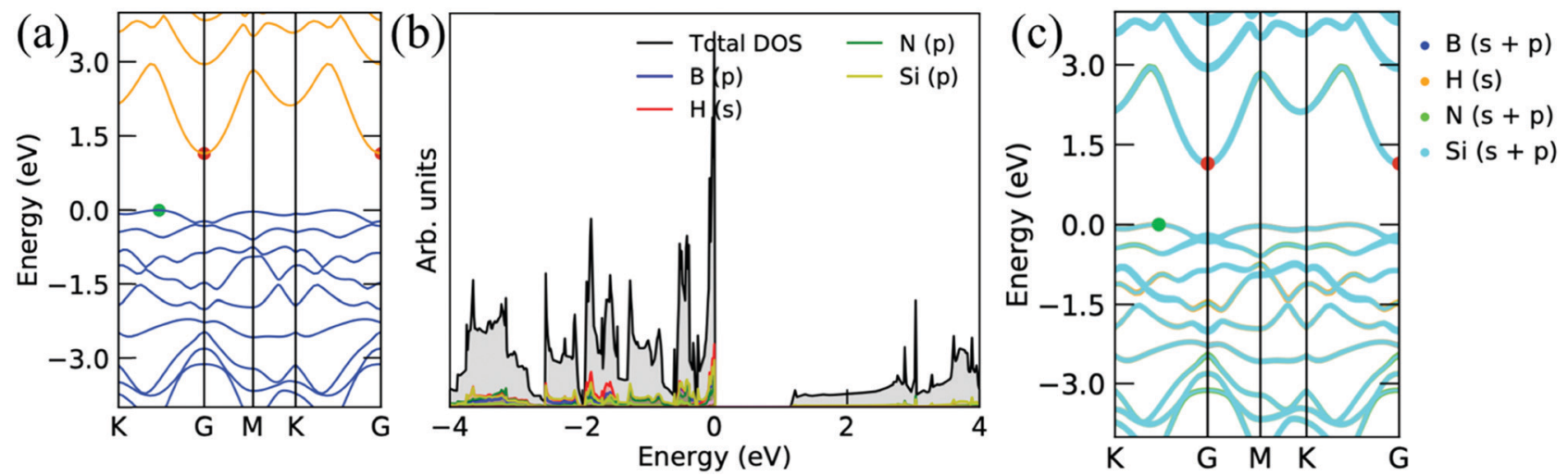

Fig. 5 Electronic band structures and corresponding projected density of states of $\mathrm{Si}_{2} \mathrm{BN}$ surface with single side hydrogenation. 

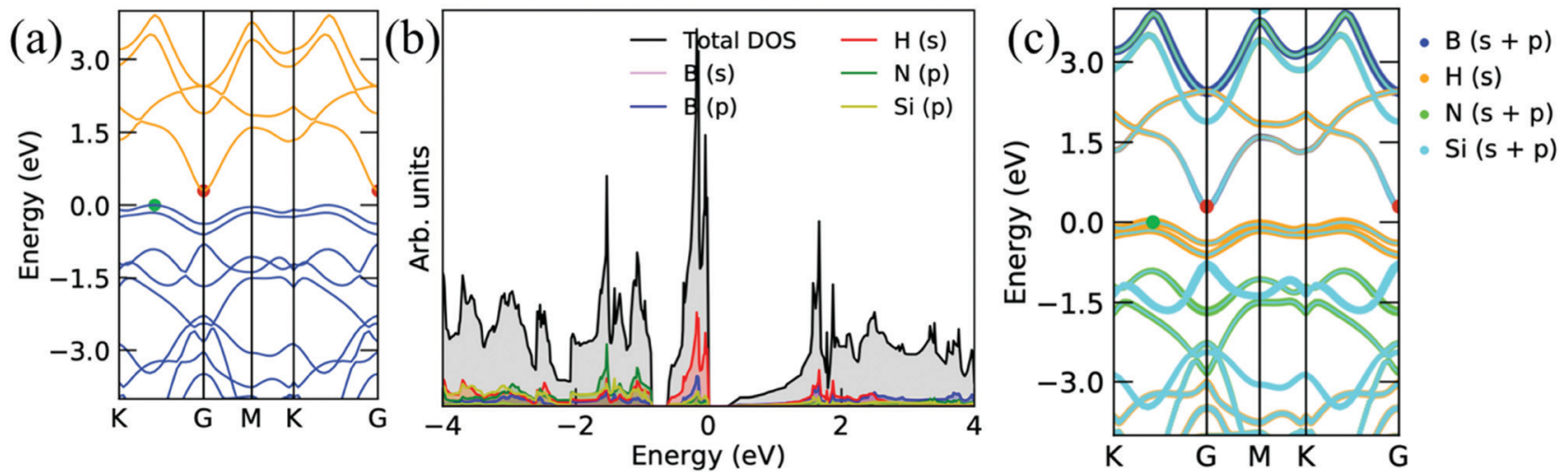

Fig. 6 Electronic band structures and corresponding projected density of states of $\mathrm{Si}_{2} \mathrm{BN}$ surface with the hydrogenation of the Si atom on both sides.
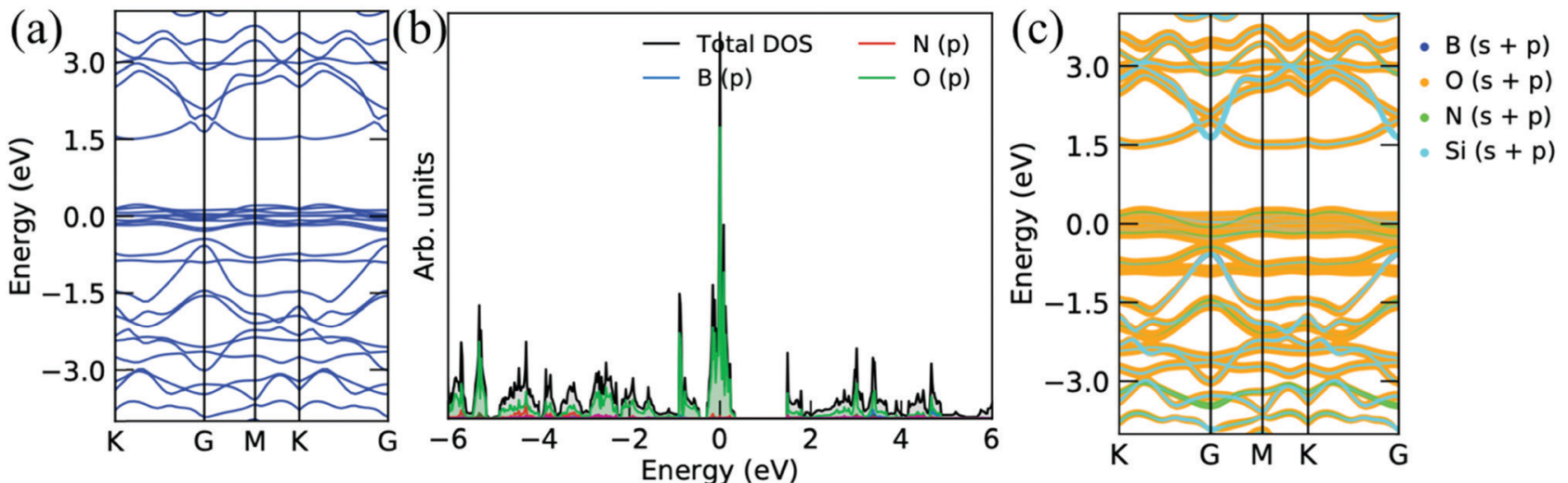

Fig. 7 Electronic band structures and corresponding projected density of states of the $\mathrm{Si}_{2} \mathrm{BN}$ surface with full oxidation.
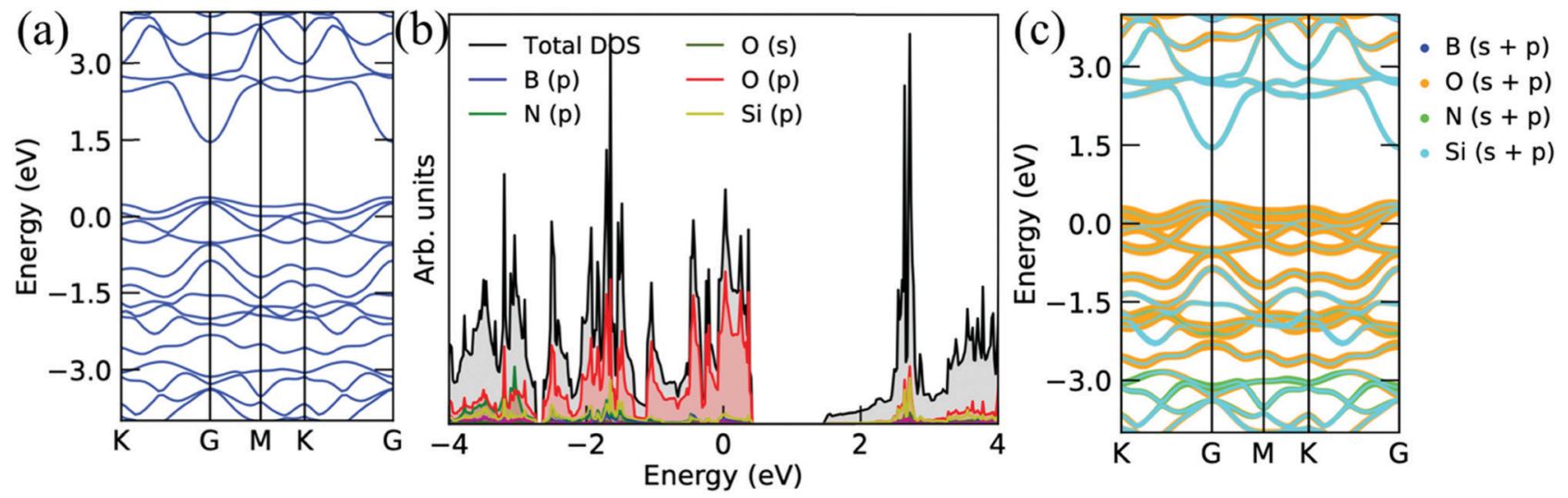

Fig. 8 Electronic band structures and corresponding projected density of states of the $\mathrm{Si}_{2} \mathrm{BN}$ surface with single side oxidation.

of electronic states is reflecting in the thermo power graph, which is described below.

\section{Thermoelectric properties}

We investigated the thermoelectric properties such as the Seebeck coefficient, electrical conductivity, thermal conductivity, power factor and figure of merit as a function of the chemical potential of pristine, hydrogenated, and oxidized $\mathrm{Si}_{2} \mathrm{BN}$ monolayer states via the Boltzmann transport theory. In addition, the constant relaxation time approximation with $\tau=10^{-14} \mathrm{~s}$ is used to calculate the electrical and thermal conductivity and power factor. The Seebeck coefficient's physical meaning is the magnitude of the potential difference generated at a specific temperature difference $\Delta T$. We calculated the Seebeck coefficient for pure $\mathrm{Si}_{2} \mathrm{BN}$, after full 

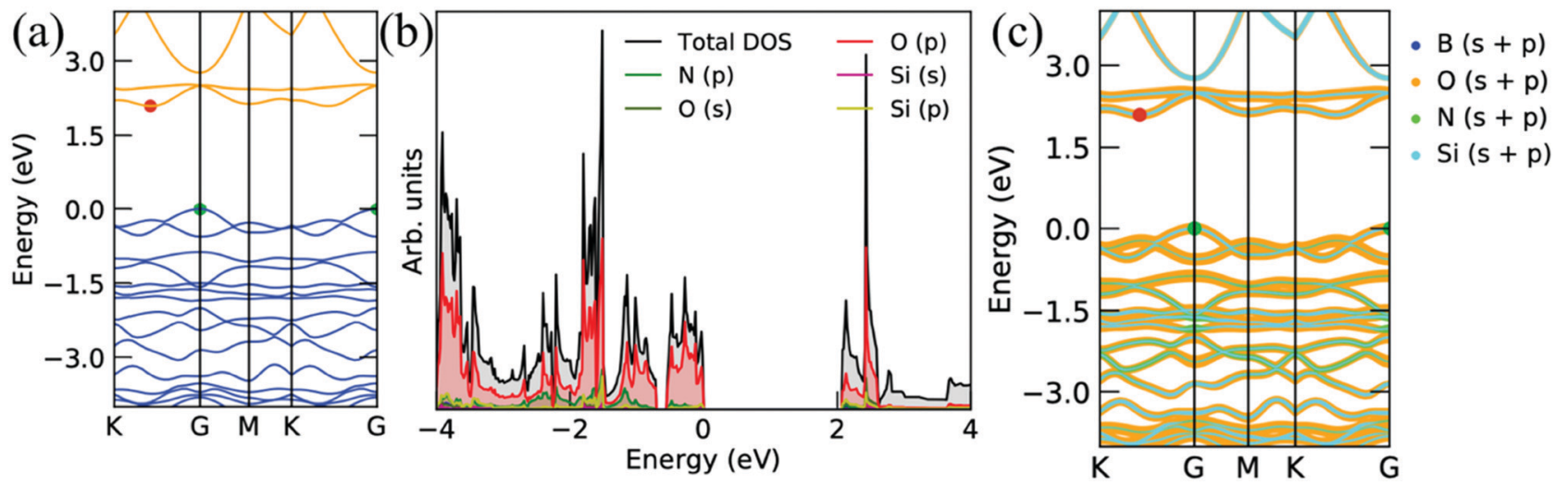

Fig. 9 Electronic band structures and corresponding projected density of states of $\mathrm{Si}_{2} \mathrm{BN}$ surface with the $\mathrm{Si}$ atom oxidation on both sides.

hydrogenation, hydrogenation of the $\mathrm{Si}$ atom on both sides, hydrogenation of each atom on a single side, full oxidation, oxidation of the $\mathrm{Si}$ atom on both sides, and after oxidation of each atom on a single side as a function of chemical potential, as shown in Fig. 10. The maximum Seebeck coefficient of seven $\mathrm{Si}_{2} \mathrm{BN}$ forms are the peak values of $\mathrm{Si}_{2} \mathrm{BNP}, \mathrm{Si}_{2} \mathrm{BNH}, \mathrm{Si}_{2} \mathrm{BNH}_{\mathrm{S}}$, $\mathrm{Si}_{2} \mathrm{BNH}_{\mathrm{S}}, \mathrm{Si}_{2} \mathrm{BNO}, \mathrm{Si}_{2} \mathrm{BNO}_{\mathrm{Si}}$, and $\mathrm{Si}_{2} \mathrm{BNO}_{\mathrm{S}}$ are 153.39, 81.17, $407.26,1815.07,1726.57,2870.52$ and $1652.82 \mu \mathrm{V} \mathrm{K}^{-1}$ at room temperature, respectively. The difference in values of the Seebeck coefficient are strongly reflected in the electronic band structures. The Seebeck coefficient of pristine $\mathrm{Si}_{2} \mathrm{BN}$ is very small compared with that of the oxidized system. Oxidation has the maximum Seebeck coefficient due to a large number of electronic states present near $E_{\mathrm{F}}$. The Seebeck coefficient of $\mathrm{Si}_{2} \mathrm{BNH}_{\mathrm{Si}}$ is small compared with that of $\mathrm{Si}_{2} \mathrm{BNO}_{\mathrm{Si}} \cdot \mathrm{Si}_{2} \mathrm{BNH}_{\mathrm{Si}}$ has more significant Seebeck coefficient than the $\mathrm{Si}_{2} \mathrm{BNP}$ system, which is caused by the sharp electronic states at the top of the valence band. The functionalized $\mathrm{Si}_{2} \mathrm{BN}$ surface has a large Seebeck coefficient, which means that it performs better for thermoelectric devices.

Fig. 11 shows the electrical conductivity of pristine, hydrogenated, and oxidized $\mathrm{Si}_{2} \mathrm{BN}$ surfaces as a function of chemical potential. The electrical conductivity is strongly dependent on

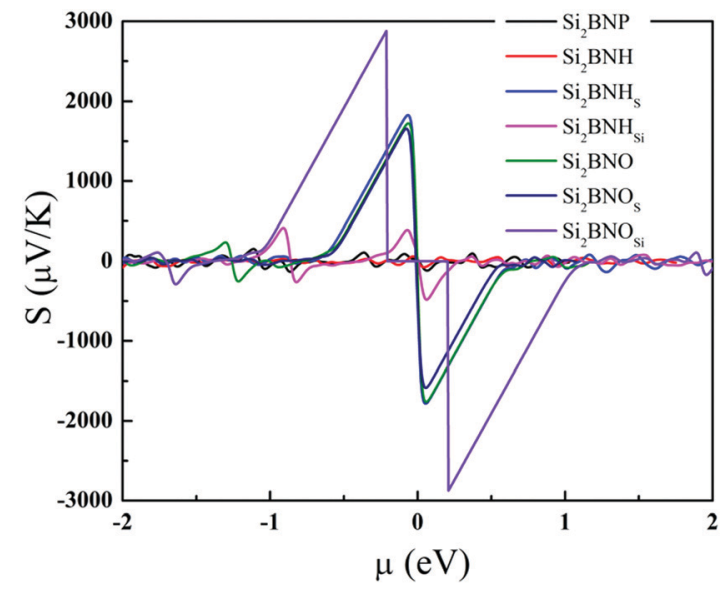

Fig. 10 Seebeck coefficient of the functionalized $\mathrm{Si}_{2} \mathrm{BN}$ monolayer as a function of the chemical potential $(\mu)$ at $300 \mathrm{~K}$. the electronic band lines, which are present on the conduction band's top and bottom. The flat band lines have large effective mass and vice versa because carrier mobility is invasively proportional to the effective mass and electrical conductivity is directly proportional to the carrier mobility (i.e., $\sigma \propto \mu$ ). The electrical conductivity of $\mathrm{Si}_{2} \mathrm{BNP}$ is much higher than that of $\mathrm{Si}_{2} \mathrm{BNH}_{\mathrm{Si}}$ since the large band gap inhibits the potential transition of the electrons. Also, at room temperature, the electrical conductivity of $\mathrm{Si}_{2} \mathrm{BNH}$ is relatively high in the positive and negative chemical potential sides compared to that of $\mathrm{Si}_{2} \mathrm{BNO}$. However, the Seebeck coefficient and electrical conductivity are inversely connected: as the doping concentration increases, the electrical conductivity increases, and the Seebeck coefficient decreases. Therefore, there exists a trade-off between the electrical conductivity and Seebeck coefficient to achieve a highpower factor. Comparing Fig. 10 with Fig. 11, we find that at the chemical potential where the Seebeck coefficient reaches the peak value, the electrical conductivity is very small and vice versa. This contradictory behavior suggests that there must be a trade-off between the Seebeck coefficient $(S)$ and the electrical conductivity $(\sigma)$. The power factor $\left(S^{2} \sigma\right)$ can be maximized at a particular doping level or carrier concentration.

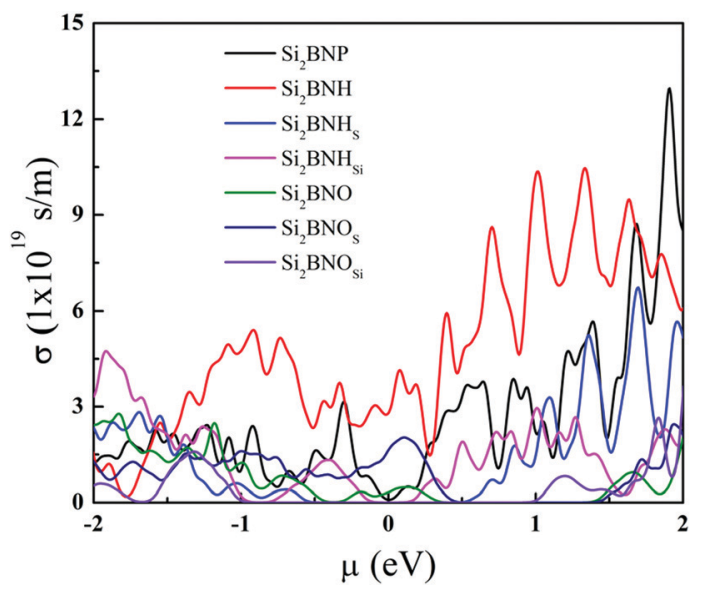

Fig. 11 Electrical conductivity of various systems as a function of chemical potential at $300 \mathrm{~K}$. 


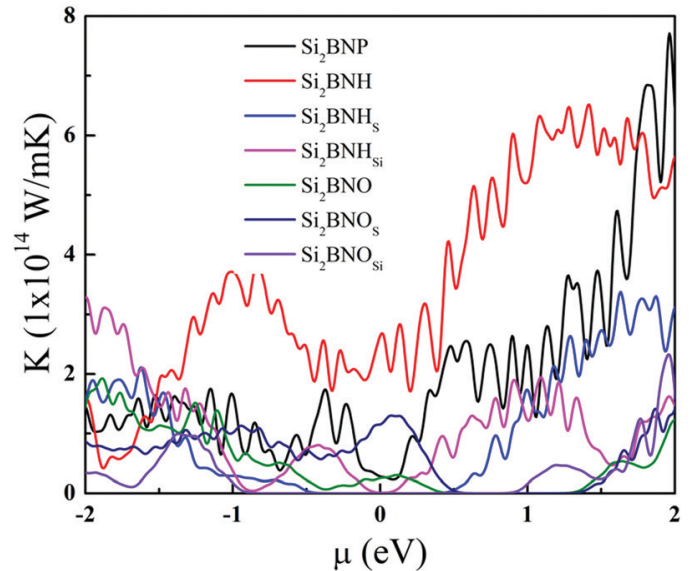

Fig. 12 Variation of thermalconductivity as a function of chemical potential at $300 \mathrm{~K}$

Next, we discussed the thermal conductivity, which is also an essential parameter for TE materials. Therefore, we have also computed the thermal conductivity response as a function of chemical potential compared with other structures at a constant relaxation time $\tau \approx 10^{-14} \mathrm{~s}$ (see Fig. 12). Efficient thermoelectric is made of materials with low thermal conductivity. ${ }^{36}$ The thermal conductivity $(k)$ comprises the electronic (electron and hole transporting heat) and phonon (phonon traveling through the lattice) contributions. We calculated the electronic part (in the form of $\tau$ ) using the constant relaxation time approximation as before as a function of $\mu$ at $300 \mathrm{~K}$ temperature, and the results are given in Fig. 12. Minimum values of $k_{\mathrm{e}}$ are seen in the chemical potential ranges between -1 and $1 \mathrm{eV}$ for all the cases present in the regions where the investigated materials will give its maximum efficiency. The maximum values of the electronic thermal conductivity at the chemical potential ranges stated before are shown in Table 1.

Now, let us turn to the power factor $\left(P=S^{2} \sigma\right)$, which is crucial in characterizing the thermoelectric potential of materials. It appears in the numerator of the expression for the figure of merit $Z T=S^{2} \sigma T / k$. One usually tries to increase the value of $P$ as much as one can without increasing the total thermal conductivity $k$. The efficiency of thermoelectric materials could be enhanced by increasing the power factor. Fig. 13 displays the power factor with constant relaxation time as a chemical

Table 1 The maximum values of the electronic thermal conductivity at the chemical potential range of -2 to $2 \mathrm{eV}$

\begin{tabular}{lll}
\hline Structure & Defect & $\begin{array}{l}\text { Maximum thermal } \\
\text { conductivity }\left(\mathrm{W} \mathrm{mK} \mathrm{mK}^{-1}\right)\end{array}$ \\
\hline Pristine & $\mathrm{Si}_{2} \mathrm{BNP}$ & 7.70 \\
$\mathrm{Hyd}$ & & \\
& $\mathrm{Si}_{2} \mathrm{BNH}_{\mathrm{S}}$ & 3.34 \\
& $\mathrm{Si}_{2} \mathrm{BNH}$ & 6.51 \\
& $\mathrm{Si}_{2} \mathrm{BNH}_{\mathrm{Si}}$ & 3.27 \\
& & \\
Oxy & $\mathrm{Si}_{2} \mathrm{BNO}_{\mathrm{S}}$ & 1.29 \\
& $\mathrm{Si}_{2} \mathrm{BNO}$ & 1.92 \\
& $\mathrm{Si}_{2} \mathrm{BNO}_{\mathrm{Si}}$ & 2.31
\end{tabular}

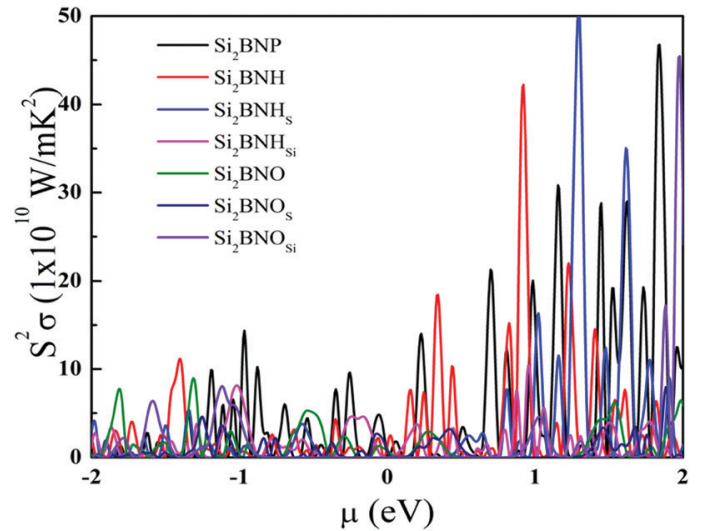

Fig. 13 Variation of the power factor as a function of chemical potential at $300 \mathrm{~K}$.

potential function relative to the Fermi level at room temperature. Due to the trade-off between electrical conductivity and Seebeck coefficient, the power factor always reaches a peak value at a low chemical potential. The power factor is minimum near the Fermi level. The maximum value of the power factor is maximum near $\mu=0.9$ to $2.0 \mathrm{eV}$ (i.e., n-type) for the hydrogenated and oxidized $\mathrm{Si}_{2} \mathrm{BN}$ monolayer.

The electronic figure of merit, $Z T_{\mathrm{e}}$, depends on the ratio of electronic and thermal conductivity, and it is also proportional to the square of the Seebeck coefficient. Fig. 14 summarizes the $Z T_{\mathrm{e}}$ dependence of the chemical potential $(\mu)$ at $300 \mathrm{~K}$. This quantity investigates the thermoelectric efficiency of materials. The highest value of $Z T$ are found to be 0.45 for $\mathrm{Si}_{2} \mathrm{BNP}, 0.21$ $\left(\mathrm{Si}_{2} \mathrm{BNH}\right), 0.80\left(\mathrm{Si}_{2} \mathrm{BNH}_{\mathrm{Si}}\right), 0.99\left(\mathrm{Si}_{2} \mathrm{BNH}_{\mathrm{S}}\right), 0.99\left(\mathrm{Si}_{2} \mathrm{BNO}\right), 0.99$ $\left(\mathrm{Si}_{2} \mathrm{BNO}_{\mathrm{Si}}\right)$, and $0.95\left(\mathrm{Si}_{2} \mathrm{BNO}_{\mathrm{S}}\right)$. The $\mathrm{ZT}$ value in the present study is higher than most of the $2 \mathrm{D}$ materials. Previously reported values of $Z T$ are found to be 0.08 for a single layer of graphene, ${ }^{37}$ and 0.12 for $\beta-, 0.03$ for $\alpha-, 0.05$ for $(6,6,12)$-, and 0.17 for $\gamma$-graphyne, ${ }^{38,39} 0.38$ in CP monolayer, ${ }^{40} 0.75$ for arsenene monolayer ${ }^{41} 0.78$ for antimonene monolayer, ${ }^{41} 1.02$ for boron monochalcogenide ${ }^{42}$ and 1.13 for holey graphene. ${ }^{43}$ In our case, we found that the value of figure of merit $Z T$ is 0.99 for the $\mathrm{Si}_{2} \mathrm{BNO}$ monolayer. Our results provide a new

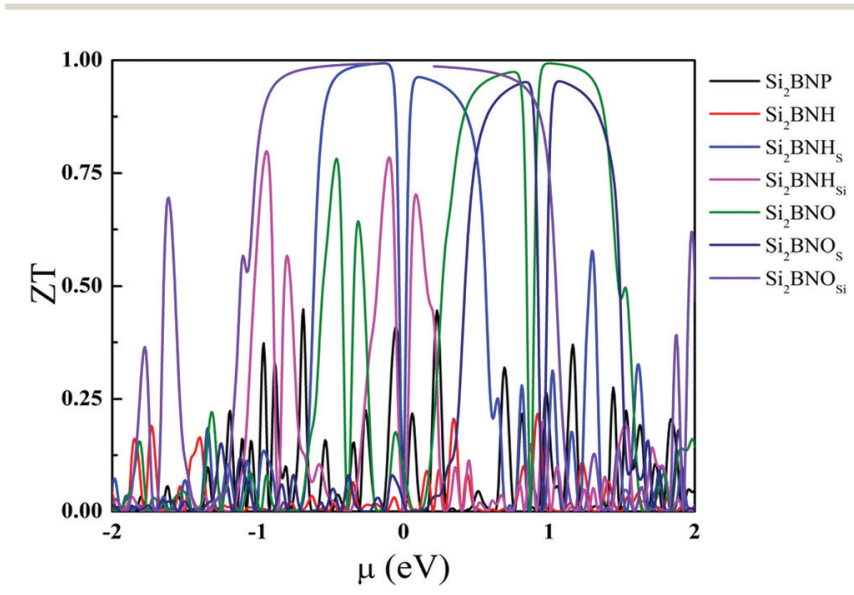

Fig. 14 Calculated $Z T$ values as a function of the chemical potential at $300 \mathrm{~K}$ 
approach for enhancing the thermoelectric performance and display the improvement of the $2 \mathrm{D}$ layered material for the applications in renewable energy.

\section{Conclusions}

In conclusion, we have systematically investigated the structural, electronic, and thermoelectric properties of pristine $\mathrm{Si}_{2} \mathrm{BN}$ and its hydrogenated and oxidized surface by firstprinciples calculations. The optimized structures are energetically favorable structures due to the negative binding energy. The electronic properties can also be tuned by the hydrogenation and oxidation of the $\mathrm{Si}_{2} \mathrm{BN}$ monolayer, transforming from the metallic to the semiconducting nature. The band gap varies from 0 to $2.10 \mathrm{eV}$ and can be achieved by the hydrogenation and oxidation of the $\mathrm{Si}_{2} \mathrm{BN}$ surface. Significant changes can be achieved by the hydrogenation and oxidation of the $\mathrm{Si}_{2} \mathrm{BN}$ monolayer, which can transform the planar structure to nonplanar structure. The pristine $\mathrm{Si}_{2} \mathrm{BN}$ monolayer has a very low thermoelectric performance. With the hydrogenation and oxidation of the $\mathrm{Si}_{2} \mathrm{BN}$ monolayer, the $\mathrm{H}$ and $\mathrm{O}$ atoms are strongly hybridized with the $\mathrm{Si}, \mathrm{B}$, and $\mathrm{N}$ atoms in the $\mathrm{Si}_{2} \mathrm{BN}$ surface due to which a band gap can be achieved, and enhancing the thermoelectric performance. The electronic figure of merit of the oxidized surface of the $\mathrm{Si}_{2} \mathrm{BN}$ monolayer enhanced up to 0.99. The theoretical investigations provide a viable strategy to improve the thermoelectric performance of the $\mathrm{Si}_{2} \mathrm{BN}$ system and can be potentially extended to other smart 2D materials' thermoelectric performance.

\section{Conflicts of interest}

There are no conflicts to declare.

\section{Acknowledgements}

D. S. and R. A. thank Olle Engkvists Stiftelse (198-0390), Carl Tryggers Stiftelse for Vetenskaplig Forskning (CTS: 18:4) and Swedish Research Council (VR-2016-06014) for financial support. S. K. G. thanks the Science and Engineering Research Board (SERB), Department of Science and Technology (India) and the Russian Foundation for Basic Research (Russia) for the financial support grant numbers: YSS/2015/001269 and INT/ RUS/RFBR/IDIR/P-6/2016.SNIC and HPC2N are acknowledged for providing the computing facilities.

\section{References}

1 J. C. Peltier, Ann. Chim. Phys., 1834, 56, 371-386.

2 A. Yadav, P. C. Deshmukh, K. Roberts, N. M. Jisrawi and S. R. Valluri, J. Phys. Commun., 2019, 3, 105001.

3 W. Thomson, Proc. R. Soc. Edinburgh, 1857, 21, 123-171.

4 G. J. Snyder and E. S. Toberer, Nat. Mater., 2008, 7, 105.

5 T. J. Zhu, Y. T. Liu, C. G. Fu, J. P. Heremans, G. J. Snyder and X. B. Zhao, Adv. Mater., 2017, 29, 1605884.
6 H. S. Kim, W. S. Liu and Z. F. Ren, Energy Environ. Sci., 2017, 10, 69.

7 J. P. Fleurial, A. Borshchevsky, T. Caillat and R. Ewell, In IECEC-97 Proceedings, 1997, vol. 2, pp. 1080-1085.

8 Q. Wang, R. Quhe, Z. Guan, L. Wu, J. Bi, P. Guan and P. Lu, RSC Adv., 2018, 8, 21280-21287.

9 M. S. Dresselhaus, G. Chen, M. Y. Tang, R. Yang, H. Lee, D. Wang, Z. Ren, J. P. Fleurial and P. Gogna, Adv. Mater., 2007, 19, 1043-1053.

10 W. G. Zeier, J. Schmitt, G. Hautier, U. Aydemir, Z. M. Gibbs, C. Felser and G. J. Snyder, Nat. Rev. Mater., 2016, 1, 16032.

11 D. Singh, S. Kansara, S. K. Gupta and Y. Sonvane, J. Mater. Sci., 2018, 53, 8314-8327.

12 C. Gaynerand and K. K. Kar, Prog. Mater. Sci., 2016, 83, 330. 13 W. Liu, X. Yan, G. Chen and Z. Ren, Nano Energy, 2012, 1, 42. 14 N. Peranio, O. Eibl, S. Babler, K. Nielsch, B. Klobes, R. Hermann, M. Daniel, M. Albrecht, H. Gorlitz and V. Pacheco, et al., Phys. Status Solidi A, 2016, 213, 739.

15 W. Liu, Q. Jie, H. S. Kim and Z. Ren, Acta Mater., 2015, 87, 357.

16 J. Matsuno, J. Fujioka, T. Okuda, K. Ueno, T. Mizokawa and T. Katsufuji, Sci. Technol. Adv. Mater., 2018, 19, 899.

17 L. E. Bell, J. Sci., 2008, 321, 1457.

18 J. P. Perdew, K. Burke and M. Ernzerhof, Phys. Rev. Lett., 1996, 77, 3865.

19 J. P. Perdew, K. Burke and Y. Wang, Phys. Rev. B: Condens. Matter Mater. Phys., 1996, 54, 16533.

20 G. Kresse and J. Furthmuller, Phys. Rev. B: Condens. Matter Mater. Phys., 1996, 54, 11169.

21 G. Kresse and J. Furthmuller, Comput. Mater. Sci., 1996, 6, 15-50.

22 G. Kresse and D. Joubert, Phys. Rev. B: Condens. Matter Mater. Phys., 1999, 59, 1758.

23 H. J. Monkhorst and J. D. Pack, Phys. Rev. B: Solid State, 1976, 13, 5188.

24 K. Momma and F. Izumi, J. Appl. Crystallogr., 2011, 44, 1272-1276.

25 J. Heyd, G. E. Scuseria and M. Ernzerhof, J. Chem. Phys., 2003, 118, 8207.

26 W. Li, L. Lindsay, D. Broido, D. A. Stewart and N. Mingo, Phys. Rev. B: Condens. Matter Mater. Phys., 2012, 86, 174307.

27 D. Singh, S. K. Gupta, Y. Sonvane and R. Ahuja, Int. J. Hydrogen Energy, 2017, 42, 22942.

28 V. Shukla, R. B. Araujo, N. K. Jena and R. Ahuja, Nano Energy, 2017, 41, 251.

29 D. Singh, S. K. Gupta, Y. Sonvane, T. Hussain and R. Ahuja, Phys. Chem. Chem. Phys., 2018, 20, 21716.

30 A. N. Andriotis, E. Richter and M. Menon, Phys. Rev. B, 2016, 93, 081413.

31 N. R. Glavin, R. Rao, V. Varshney, E. Bianco, A. Apte, A. Roy and P. M. Ajayan, Adv. Mater., 2020, 32, 1904302.

32 O. U. Akturk, V. Ongun, O. Zcelik and S. Ciraci, Phys. Rev. B: Condens. Matter Mater. Phys., 2015, 91, 235446.

33 S. Cahangirov, H. Sahin, G. Le Lay and A. Rubio, Introduction to the Physics of Silicene and other 2D Materials, Springer, 2016, vol. 930.

34 C. Kamal and M. Ezawa, Phys. Rev. B: Condens. Matter Mater. Phys., 2015, 91, 085423. 
35 W. Xu, L. Zhu, Y. Cai, G. Zhang and B. Li, J. Appl. Phys., 2015, 117, 214308.

36 M. Onoue and T. Oguchi, J. Phys. Soc. Jpn., 2008, 77, 054706. 37 A. H. Reshak, S. A. Khan and S. Auluck, J. Mater. Chem. C, 2014, 2, 2346-2352.

38 H. Sevinçli and C. Sevik, Appl. Phys. Lett., 2014, 105, 223108.

39 M. Ali, X. Pi, Y. Liu and D. Yang, Nanotechnology, 2017, 29, 045402.
40 D. Singh, S. Kansara, S. K. Gupta and Y. Sonvane, J. Mater. Sci., 2018, 53, 8314-8327.

41 S. Sharma, S. Kumar and U. Schwingenschlogl, Phys. Rev. Appl., 2017, 8, 044013.

42 P. Mishra, D. Singh, Y. Sonvane and R. Ahuja, Sustainable Energy Fuels, 2020, 4, 2363-2369.

43 D. Singh, V. Shukla and R. Ahuja, Phys. Rev. B, 2020, 102, 075444 . 